\title{
Accelerated Failure Time Model to Explore the Perception Response Times of Drivers in Simulated Car-Following Scenarios
}

\author{
Yingshi Guo, ${ }^{1}$ Zhi Zhang $\mathbb{D}^{1},{ }^{1}$ Wei Yuan, ${ }^{1}$ Chang Wang, ${ }^{1}$ Fuwei Wu, ${ }^{1}$ and Zhuofan Liu ${ }^{2}$ \\ ${ }^{1}$ School of Automobile, Chang'an University, Xi'an 710064, China \\ ${ }^{2} X i$ 'an University of Posts and Telecommunications, Xi'an 710061, China \\ Correspondence should be addressed to Zhi Zhang; zhangzhi@chd.edu.cn
}

Received 20 March 2020; Revised 20 May 2020; Accepted 30 June 2020; Published 23 July 2020

Academic Editor: Jaeyoung Lee

Copyright (c) 2020 Yingshi Guo et al. This is an open access article distributed under the Creative Commons Attribution License, which permits unrestricted use, distribution, and reproduction in any medium, provided the original work is properly cited.

In the development of effective rear-end collision alarm systems, understanding the factors that influence the perception response times (PRT) of drivers is important for the design of a reasonable lead time for the warning (or intervention) of likely collisions. Previous studies have proposed different approaches for examining the impact of situational or individual factors on the PRT of drivers. However, unobserved heterogeneity has not been considered and neither has a duration-modeling approach been used, resulting in a lack of accurate estimation. The purpose of the present study was to explore the effect of the driving situation and individual differences on the PRT of drivers while also considering unobserved heterogeneity. A total of 101 participants were exposed to different levels of secondarily cognitive load and situational urgency in simulated d scenarios. Several accelerated failure time (AFT) duration models, both with and without heterogeneity, were developed to model the PRT of drivers, while factors related to driving situation and individual differences were incorporated. The results indicate that influential factors include age, working memory capacity (WMC), cognitive load, and initial time headway exerted significant effects on the PRT of drivers. The hazard rate changed by $14.4 \%, 22.6 \%$, and $7.5 \%$ when age, cognitive load, and initial time headway changed by one unit, respectively. Furthermore, the hazard rate decreases by more than $20 \%$ for individuals with higher WMC compared with baseline individuals. These results suggest that the AFT model that considers unobserved heterogeneity can provide a more accurate estimation of the PRT compared to other duration models. These findings can be expected to provide a further understanding of drivers' braking behaviors, which will contribute to the development of advanced driving assistant systems as well as safety assessments of in-vehicle information and communication technologies.

\section{Introduction}

The World Health Organization (WHO) reported that the number of annual road-traffic deaths has reached 1.35 million and that road-traffic injuries have become the leading killer of people aged 5-29 years, and the burden is disproportionately borne by traffic users of developing countries [1]. A survey conducted in the Chinese city of Shanghai showed that rear-end collisions account for $20 \%$ of all crash types and $49 \%$ of elevated expressway crashes [2]. To avoid rear-end collisions, rear-end collision alarm systems have been proposed to warn or intervene in driver behaviors to avert possible collisions. For instance, a sudden brake of the lead vehicle can be informed in advance, or the brake is automatically activated when the driver cannot perceive the hazard in time. With this regard, understanding factors that influence the perception response time (PRT) of drivers in car-following driving scenarios is of great importance. This is due to the significant safety and operational impacts attributable to a reasonable lead time for the warning or intervention of possible collisions for the development of effective rear-end collision alarm systems.

With regard to human information processing, PRT represents the time required to perceive, interpret, decide, and initiate a response to a stimulus [3]. In the case of carfollowing driving, PRT is commonly referred to as the time between the activation of the lead vehicle's brake lamp and the initial application of pressure to the brake pedal by the 
following car [4]. Situational factors, such as the initial time headway, the deceleration rate of lead vehicle, and secondarily cognitive load, were recognized as major factors that influence drivers' PRT in a car-following event [5-7]. In general, the PRT increased with increasing initial time headway or secondarily cognitive load but decreased with increasing deceleration rate of the lead vehicle. One explanation is that drivers' response to a lead vehicle braking is driven by two sources of sensory evidence: visual looming and brake light. As the time headway decreased, the visual looming accumulated consistently to some extent, and the drivers' response to a lead vehicle braking is triggered. This has been considered as an automatic process that does not involve the cognitive control system. Alternatively, drivers' braking behaviors could also be driven by the brake light of a lead vehicle via top-down mechanisms. This process was reported to be dependent on cognitive control resources and could thus be affected by cognitive load [8]. For example, when drivers are cognitively distracted, their PRT increases because of inadequate cognitive resources.

Individual differences in cognitive resources were also attributable to variations of PRT. As a major component of cognitive resource, the working memory capacity (WMC) can be identified as how well individuals can control their attention and maintain information in an active and quickly retrievable state $[9,10]$. Evidence indicates that WMC is closely related to effective multitasking ability in shooting behavior [11], academic performance [12], and a simulated breakfast-making task [13]. In studies of driving performances, Johannsdottir and Herdman [14] found that WMC played an important role in supporting driver situation awareness for traffic located in both the forward view and the rear view. Ross et al. [15] found higher WMC to be related to better performance in a lane-change task in terms of mean deviation in the lane-change path, lanechange initiation, and percentage of correct lane changes. Wood et al. [16] explored the relationship between WMC and hazard perception under dual task conditions and found that low WMC individuals had poorer hazard perception performance when they were involved in the secondary task, and these performance decrements were mirrored in decreases of mean fixation durations on the hazard. In a recent study, Louie and Mouloua [17] studied the reaction times of drivers by analyzing their responses when the traffic lights appeared yellow and showed that high WMC could impair the effects of distraction tasks on reaction time. Despite the vast availability of literature on the effects of drivers WMC on their driving performances, only one car-following study examined the relationship between WMC and reaction time to lead vehicle's braking event. The results showed that a majority of drivers prolonged their braking reaction time under the influence of secondarily cognitive load, but several high WMC drivers were even not affected by such secondarily cognitive tasks [7]. In addition to the influential factors mentioned above, driver age and gender were also found to significantly affect PRT [18]. However, because of a large range of $0.5-10 \mathrm{~s}$ in PRT values [19], these observed factors offer limited explanation of the PRT of drivers, and unobserved heterogeneity was not considered in these studies. Consequently, these studies did not provide an accurate estimation of risk factors.
Recently, several scholars applied duration models to explore braking behavior of drivers. As a probabilistic approach, hazard-based duration models are well suitable to analyze time-related data [20]. Duration models have been applied to describe the reaction time of drivers. For example, Haque and Washington [21] used pedestrian crossing in the crosswalk and studied the effects of mobile phone conversations on driver reaction times. Choudhary and Velaga [22] used pedestrian-crossing events and road-crossing events by parked vehicles as two separate hazardous events and examined the effects of different types of phone use and different levels of complexity involved in mobile phone use on reaction times. Yadav and Velaga [23] explored the relationship between different blood alcohol concentrations and reaction times of both young and mature drivers. In these studies, parametric accelerated failure time (AFT) duration models with a Weibull distribution were applied to account for the unobserved heterogeneity arising from the repeated measures experimental design and were found to be the best fitting models for identifying significant variables that influence the reaction times of drivers.

Therefore, in this paper, a hazard-based durationmodeling approach was used to develop an estimation model of driver PRT in car-following driving scenarios. Several risk factors including situational and individual factors for drivers' braking behavior were estimated by the AFT duration models. Furthermore, unobserved heterogeneity was considered in the developed duration models. The empirical data obtained by a driving simulator are modeled by parametric hazard models with different types of distributions (Weibull, lognormal, and log-logistic distributions). Finally, the proposed models with heterogeneity were compared with traditional models without heterogeneity in terms of goodness-of-fit, and the results of the effects of situational and individual variables are discussed on the basis of the appropriate model.

\section{Method}

The adopted methodology in the present study is illustrated in Figure 1. First, simulated car-following scenarios were developed, including lead vehicle speed, initial time headway, road geometry, and traffic conditions. The participants were identified, and a computer-based WMC test and a questionnaire about demographic details were prepared. Second, experiments were conducted and the related data were collected and extracted in the laboratory. Third, the preliminary analysis was conducted on the collected data, and then the AFT models were constructed to analyze the effect of situational and individual factors on the PRT of the participants. Finally, the results were further interpreted focusing on significant explanatory variables.

2.1. Apparatus. A driving simulator method was applied to assess the effects on drivers' PRT by a large number of researchers in previous studies due to its outperformance in data collection and extraction, and safety also has to be considered [7, 21-23]. In the current study, a fix-based SILAB 5.1 driving simulator was used for data collection and extraction 


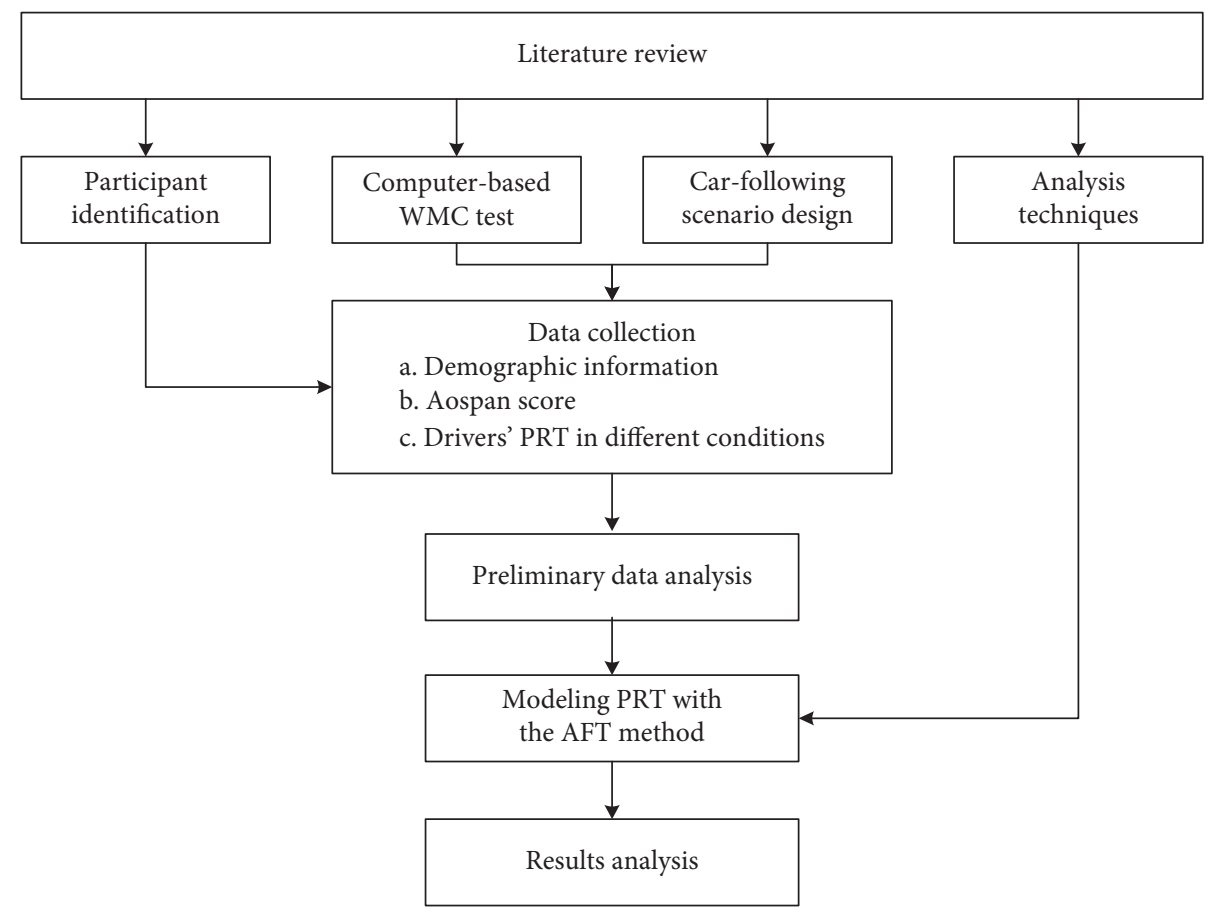

FIgure 1: The methodology adopted by the present study.

(see Figure 2). The simulator consists of three LED screens with a resolution of $1280 \times 800$ pixels, a force-feedback steering wheel, a column gear selector, an instrumental dashboard, brake and accelerator pedals, and a driver seat. Both a speedometer and tachometer are displayed at the bottom of the central screen, and two rearview mirrors are displayed at the bottom of the left and right screens. The computer system of the simulator recorded vehicle movement data and driver operation data at a sampling frequency of $60 \mathrm{~Hz}$.

2.2. Participants. A total of 101 participants (64 males and 37 females) in the age range of 20-40 years were recruited through online advertising and were offered a monetary reward. No significant age differences were found between male and female participants $(F(1,99)=0.030, p=0.863)$. Each participant had a valid driving license and had at least one year's worth of driving experience; also each had normal or corrected-to-normal vision. The participants were classified into two groups by age: young group (younger than 25 years) and mature group (25 years and above), as previously applied by many researchers [24]. About $67.3 \%$ of the participants were classified as young group ( mean $=22.18$; $\mathrm{SD}=0.863$ ) and the remaining $32.7 \%$ as mature group $($ mean $=28.97 ; \mathrm{SD}=3.077)$. None of the participants were reported to have suffered from simulator sickness. The ethical protocol of the present study was approved by the Institutional Review Board at Chang'an University.

2.3. Experimental Design. This study designed a withinsubject experiment; three tasks were used for the current study. It took about $1 \mathrm{~h}$ to finish the whole experiment for one participant.

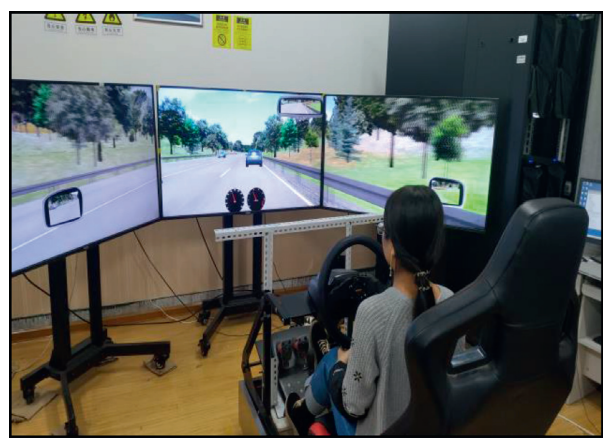

FIGURE 2: Driving simulator and experimental scenario.

2.3.1. Automated Operation Span Task. An automated operation span task (AOSPAN) was conducted to assess drivers' WMC on a computer. In this task, a total of 15 trials were presented (three trials with three to seven letters to remember); participants were required to solve a series of math problems while remembering as many unrelated letters as possible. The final score was automatically calculated, and a high score indicated good WMC. Previous studies demonstrated that the AOSPAN test showed good correlation with other WMC measures and also had both good internal consistency and test-retest reliability [25]. Otherwise, in the previous studies of driving performances, AOSPAN test was widely used to measure WMC $[7,16,26]$.

2.3.2. Secondarily Cognitive Task. One delayed digit recall task (1-back task) was adopted to induce secondarily cognitive load [15, 27]. Participants were asked to verbally repeat a list of single-digit numbers when the second digit 
was presented with a spacing of $2.25 \mathrm{~s}$ in a random order; several existing studies claimed that this task could have access to working memory resources [28, 29].

2.3.3. Simulated Driving Task. The experimental drives were conducted on a bidirectional straight expressway with a speed limit of $120 \mathrm{~km} / \mathrm{h}$ on the simulator; participants were required to perform a car-following task and respond to the braking behavior of the lead vehicle; the lead vehicle was set a fixed speed of $100 \mathrm{~km} / \mathrm{h}$. Two full stops of the lead vehicle $(0.3 \mathrm{~g}$ and $0.6 \mathrm{~g}$ of braking deceleration rate) with brake lights on were executed randomly, and participants were orally informed to drive within the specified following distance during the drive (about 30-60 m); accordingly, the range of time headway was about 1-2 s. However, in actual tests, the time headway between two vehicles varied in a larger range. To help the subjects determine the car-following distance, a guardrail was set on the roadside, and a distance of $5 \mathrm{~m}$ between the two bars was set as a reference.

2.4. Experimental Procedure. First, each participant was asked to complete a demographic information questionnaire, which asked for age, gender, and driving experience. Second, a computer-based test was performed for assessing all participants' WMC. Finally, after finishing a $5 \mathrm{~min}$ training drive to familiarize with the driving simulator, each participant was subjected to two experimental drives (with baseline load and with secondarily cognitive load). These two drives were conducted in a counterbalanced order; that is, about one half of the participants drove without secondarily cognitive load first, while the other half drove with secondarily cognitive load first.

2.5. Data Acquisition. A total of $404(101 * 4)$ simulated carfollowing scenarios were created. Further examination showed that 13 of them involved the release of accelerator pedal before the lead vehicle began to brake, and 31 of them involved exceeding the preset following distance range when the lead vehicle began to brake. Therefore, the data for the remaining 360 scenarios were analyzed. In the current study, since the driving simulator was not able to directly record the PRT value, according to previous studies, it was calculated as the time between the activation of the lead vehicle's brake lamp and the initial application of pressure onto the brake pedal $[2,4]$.

2.6. Accelerated Failure Time Models. The key variable in hazard-based duration models is the length of time elapsed before an event takes place. In the current study, an event is defined as the detection of lead vehicle's braking behavior; the PRT is defined as the duration between the trigger of the lead vehicle's brake lamp and the initiation of the participant's brake pedal. Let $T$ be a continuous and nonnegative random variable representing PRT, and $f(t)$ and $F(t)$ are the probability density function and cumulative distribution function of $T$, respectively. Both are related as follows:

$$
f(t)=\frac{d F(t)}{d t}=\frac{d p(T<t)}{d t} .
$$

Let $S(t)$ denote the survivor function, which is the probability that the duration variable (PRT) is greater than or equal to time $t$. In this study, this is the probability that the driver fails to detect the lead vehicle's braking behavior or fails to initiate the brake pedal. It can be described as follows:

$$
S(t)=p(T \geq t)=1-F(t) .
$$

Let $h(t)$ denote the hazard function, which is the conditional probability that an event occurs between times $t$ and $t+d t$, given that the event has survived until time $t$. In this study, the probability of the detection of the lead vehicle's braking behavior increased with time and can be formulated as follows:

$$
h(t)=\lim _{\Delta t \rightarrow 0} \frac{p(t<T \leq t+\Delta t \mid T \geq t)}{\Delta t}=\frac{f(t)}{1-F(t)} .
$$

To analyze the effect of covariates in the model, it is necessary to select the appropriate approach to incorporate all covariates into the duration model. Since the parametric AFT duration model assumes that the covariates could directly accelerate the duration time in the survivor function with all covariates being zero [30], this allows the effect of the covariates to multiply the duration time by a constant. This contributes to a simpler interpretation for quantifying the effect of the covariates on the duration time. Therefore, in this study, a parametric AFT duration model was adopted to investigate driver's PRT.

Let $\ln T$ represent the natural logarithm of the duration time, which is expressed as a regression equation for duration time in AFT duration model. It can be described as follows:

$$
\ln T=\beta X+\varepsilon,
$$

where $\beta$ represents a vector of parameters estimated, $X$ represents a vector of covariates, and $\varepsilon$ represents the error term. Here, three typical duration distributions (Weibull, log-logistic, and lognormal distributions) were adopted to develop the AFT duration model. For example, when $T$ yields to Weibull distribution, the probability density function, survival function, and hazard function can be formulated as follows:

$$
\begin{aligned}
& S(t)=\exp \left[-t^{\lambda} \exp (-\lambda \beta X)\right], \\
& h(t)=\lambda t^{\lambda-1} \exp (\lambda \beta X),
\end{aligned}
$$

where $\lambda>0$ represents the scale parameter and the value of $\lambda$ determines the shape of the hazard function. If $\lambda=1$, the Weibull AFT model is reduced to the exponential AFT regression model and, consequently, the hazard function is constant. When $0<\lambda<1$, the hazard rate decreases with time; when $1<\lambda<2$, the hazard rate increases at a decreasing rate, with its shape being concave; when $\lambda>2$, the hazard rate increases with an increasing rate, and its shape is convex; lastly, when $\lambda=2$, the hazard rate increases constantly [31]. 
In this experiment, there are two types of heterogeneity with regard to the observed value of PRTs: between individuals and within individuals. The former is caused by individual differences in demographic, cognitive, and other unobserved factors $[32,33]$, while the latter is caused by repeated measures of driving performance. Since the observed PRTs are not independent, it may be correlated for one participant [21]. However, both types of heterogeneity at the individual level were not commonly considered in many traditional duration models, and the assumption of individual homogeneity of the survival distribution often leads to inaccurate estimates in the coefficient of covariates $[34,35]$.

This problem can be overcome by incorporating shared frailty into the duration model. The shared frailty model is the best solution for dealing with the unobserved heterogeneity [21]. The shared frailty model can be formulated as follows:

$$
\begin{aligned}
& h_{i j}\left(t \mid \alpha_{i}\right)=\alpha_{i} h_{i j}(t), \\
& s_{i j}\left(t \mid \alpha_{i}\right)=\exp \left\{-\alpha_{i} \int_{0}^{t} h_{i j}(x) d x\right\},
\end{aligned}
$$

where $\alpha_{i}$ represents the frailty of the $i^{\text {th }}$ individual and is assumed to be gramma-distributed. Moreover, $h_{i j}(t)$ represents the $j^{\text {th }}$ hazard function of the $i^{\text {th }}$ individual, and $S_{i j}(t)$ represents the $j^{\text {th }}$ survival function of the $i^{\text {th }}$ individual.

Therefore, the unconditional survivor function $S_{i j}^{\prime}(t)$ can be formulated as follows:

$$
s_{i j}^{\prime}(t)=\int_{0}^{\infty} \exp \left\{-\alpha_{i} \int_{0}^{t} h_{i j}(x) d x\right\} g\left(\alpha_{i}\right) d \alpha_{i}
$$

where $g\left(\alpha_{i}\right)$ represents the probability density function of $\alpha_{i}$.

To better compare the goodness-of-fit of the models and identify the best distribution in different AFT models, Akaike's information criteria (AIC) and Schwarz's Bayesian criteria (BIC) were calculated for each model with different distributions (see equations (10) and (11)). The value of AIC considers the effect of the number of parameters, and the value of BIC considers the effect of the number of parameters and sample size [36,37]. In general, the model with the smallest AIC and BIC values was selected as the final model.

$$
\begin{aligned}
& \mathrm{AIC}=-2 \ln (L)+2 k . \\
& \mathrm{BIC}=-2 \ln (L)+\ln (n) * k .
\end{aligned}
$$

\section{Results}

3.1. Preliminary Analysis of PRT. As shown in Figure 3, the minimum and maximum PRT values were represented by the lower and upper whiskers and the $25^{\text {th }}$ percentile and the median and the $75^{\text {th }}$ percentile of the PRT values were represented by the lower, middle, and upper boundaries of the box. Specifically, Figure 3(a) presents the distribution of the PRT value in both driving tasks; the median of the PRT value in baseline driving task (median $=0.983 \mathrm{~s}$ ) was shorter than that in the driving task with cognitive load $($ median $=1.683 \mathrm{~s})$. The PRT of drivers increased with cognitive load. Furthermore, Figure 3(b) shows the distribution of the PRT value in the car-following situation with different time headways; the median of the PRT value in the situation with short time headways (median $=1.300 \mathrm{~s}$ ) was slightly longer than that in the situation with long time headway $($ median $=1.133 \mathrm{~s})$. Figure $3(\mathrm{c})$ shows the distribution of the PRT value in car-following situation with different deceleration rates of lead vehicle; the median of the PRT value in the situation with $0.3 \mathrm{~g}$ of deceleration rate (median $=1.217 \mathrm{~s}$ ) was longer than that in the situation with $0.6 \mathrm{~g}$ of deceleration rate $($ median $=1.100 \mathrm{~s})$.

3.2. Models for the Effects of PRT. To further examine the effects of various factors on the PRT of drivers in car-following scenarios, Weibull AFT models with and without heterogeneity were constructed. The explanatory variables included in both models are shown in Table 1. Gender, age, and WMC were considered as individual factors, while cognitive load, initial time headway, and lead vehicle deceleration rate were considered as situational factors. Among them, initial time headway was a continuous variable, while the others were classification variables. To compare the effect of different levels of WMC on the PRT, the OSPAN score of drivers was divided into four levels: top quartile, second quartile, third quartile, and bottom quartile, which was similar to previous studies [15].

Table 2 lists the goodness-of-fit of the model with and without heterogeneity. All models achieved $p$ value $<0.001$. For each distribution, the values of AIC and BIC were lower in the model with heterogeneity compared to that without heterogeneity, suggesting that the model with heterogeneity can be better used to estimate the PRT of drivers compared with the model without heterogeneity, and the inclusion of unobserved heterogeneity was found to significantly improve the goodness-of-fit of the models. Among all models, the Weibull AFT model with heterogeneity had the lowest $\mathrm{AIC}$ and $\mathrm{BIC}$ values $(\mathrm{AIC}=402.322, \mathrm{BIC}=445.069)$; the $\mathrm{AIC}$ and BIC values decreased by 100 or more compared to the other models. Therefore, the Weibull AFT model was selected to estimate the PRT value of drivers in the present study.

The results of the Weibull AFT models with heterogeneity are summarized in Table 3 . The value of the scale parameter was estimated as 3.890 , which exceeds 2 , suggesting that the hazard of not braking pedal increases at an increasing rate as time elapses at the lead vehicle braking event. The influential factors, including age, working memory capacity, cognitive load, and initial time headway, exhibit a significant effect on the PRT of drivers. The negative coefficients of age and working memory capacity imply that an increase in the corresponding factor relates to a decrease in the hazard rate of not braking pedal and an increase in the PRT value. In contrast, the positive coefficients of cognitive load and initial time headway imply that an increase in the corresponding factor relates to an increase in the hazard 


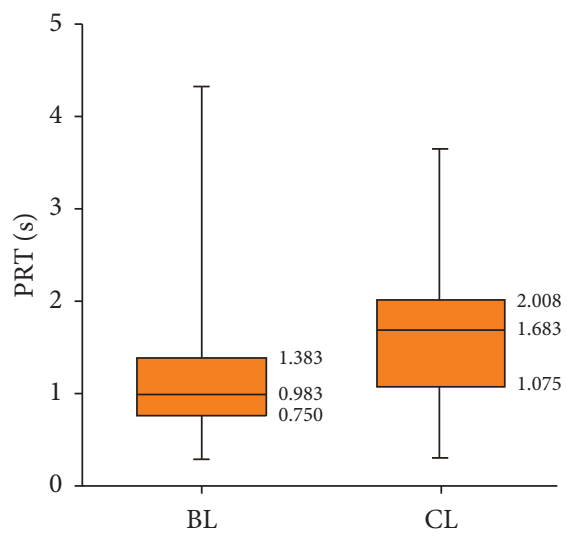

(a)

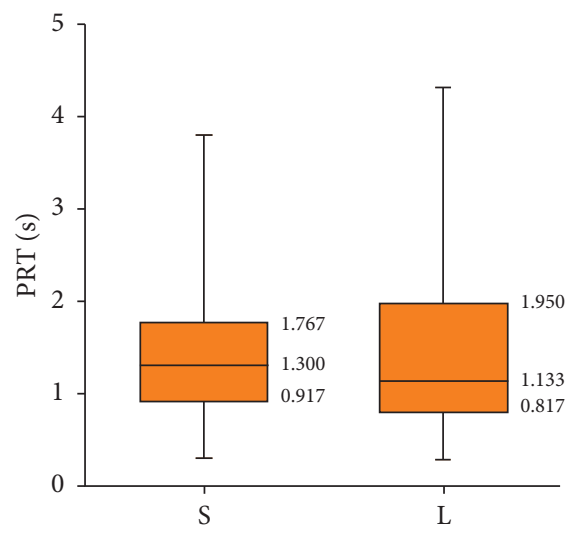

(b)

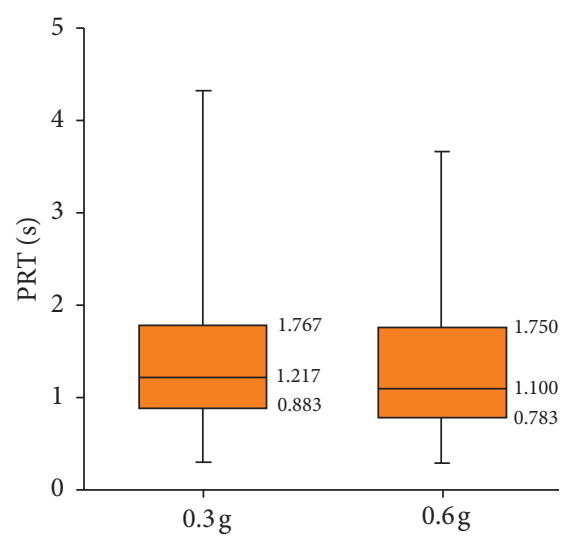

(c)

Figure 3: Effects of cognitive load, initial time headway, and lead vehicle deceleration rate. (a) Level of cognitive load. (b) Initial time headway. (c) Lead vehicle deceleration rate.

TABle 1: Definition of independent variables.

\begin{tabular}{lc}
\hline Variables & Descriptions \\
\hline Individual factors & Female: 1 ; male: 0 \\
Gender & Mature $(25-40): 1$; young $(<25): 0$ \\
Age group & Cognitive load: 1 ; baseline load: 0 \\
Level of WMC (OSPAN score) & Top quartile (63-75): 3; second quartile (57-62): $;$ third quartile $(41-56): 1 ;$ bottom quartile $(13-40): 0$ \\
\hline Situational factors & Continuous variables: mean $=1.527 ;$ std. $=0.520 ;$ range $=(0.517,2.952)$ \\
Level of cognitive load & 0.6 g: $1 ; 0.3$ g: 0 \\
Initial time headway (second) & Lead vehicle deceleration rate
\end{tabular}

TABLE 2: Comparison of fit statistics of the models.

\begin{tabular}{lcccrrr}
\hline Heterogeneity treatment & Distribution & Degrees of freedom & Log likelihood & AIC & BIC & $p$ \\
\hline \multirow{3}{*}{ Without heterogeneity } & Weibull & 10 & -274.114 & 568.227 & 607.089 & 0.000 \\
& Lognormal & 10 & -267.593 & 555.186 & 594.047 & 0.000 \\
& Log-logistic & 10 & -265.031 & 550.063 & 588.924 & 0.000 \\
\hline \multirow{3}{*}{ With heterogeneity } & Weibull & 11 & -190.161 & 402.322 & 445.069 & 0.000 \\
& Lognormal & 11 & -245.826 & 513.651 & 556.399 & 0.000 \\
& Log-logistic & 11 & -247.058 & 516.115 & 558.862 & 0.000 \\
\hline
\end{tabular}

TABle 3: Parameter estimates of Weibull AFT models with heterogeneity.

\begin{tabular}{|c|c|c|c|c|c|c|}
\hline Variable & Estimate & S.E. & $Z$-test & $95 \%$ IC & $\operatorname{Exp}(\beta)$ & Percentage change (\%) \\
\hline \multicolumn{7}{|l|}{ Gender } \\
\hline Female versus male & -0.036 & 0.099 & -0.36 & $(-0.230,0.159)$ & 0.965 & -3.5 \\
\hline \multicolumn{7}{|l|}{ Age group } \\
\hline Middle-aged versus young & -0.155 & 0.086 & $-1.80^{*}$ & $(-0.324,0.013)$ & 0.856 & -14.4 \\
\hline \multicolumn{7}{|l|}{ Level of WMC } \\
\hline The top quartile versus the bottom quartile & -0.239 & 0.122 & $-1.96^{* *}$ & $(-0.478,0.000)$ & 0.787 & -21.3 \\
\hline The second quartile versus the bottom quartile & -0.250 & 0.119 & $-2.10^{* *}$ & $(-0.484,-0.016)$ & 0.779 & -22.1 \\
\hline The third quartile versus the bottom quartile & -0.232 & 0.113 & $-2.06^{* *}$ & $(-0.453,-0.011)$ & 0.793 & -20.7 \\
\hline \multicolumn{7}{|l|}{ Level of cognitive load } \\
\hline Cognitive load versus baseline load & 0.204 & 0.043 & $4.75^{* * *}$ & $(0.120,0.288)$ & 1.226 & 22.6 \\
\hline \multicolumn{7}{|l|}{ Lead vehicle's deceleration rate } \\
\hline $0.6 \mathrm{~g}$ versus $0.3 \mathrm{~g}$ & -0.021 & 0.034 & -0.60 & $(-0.089,0.047)$ & 0.979 & -2.1 \\
\hline THW & 0.072 & 0.034 & $2.10^{* *}$ & $(0.005,0.139)$ & 1.075 & 7.5 \\
\hline Constant & 0.172 & 0.111 & 1.55 & $(-0.045,0.390)$ & & \\
\hline$\lambda$ (scale parameter) & 3.890 & 0.221 & $23.91^{* * *}$ & $(3.480,4.348)$ & & \\
\hline Theta & 1.562 & 0.287 & $2.42^{* *}$ & $(1.089,2.240)$ & & \\
\hline
\end{tabular}


rate of not braking pedal and a decrease in the PRT value. The percentage of change in the PRT value can be calculated by $(\operatorname{Exp}(\beta)-1)^{*} 100 \%$. For example, the hazard rate would change by $14.4 \%, 22.6 \%$, and $7.5 \%$ when the covariate of age, level of cognitive load, and initial time headway, respectively, change by one unit. The hazard rate would change by $21.3 \%, 22.1 \%$, and $20.7 \%$ when the covariate of working memory capacity changes from the bottom level to the top level, the second level, and the third level, respectively. Other influential factors, such as gender and lead vehicle's deceleration rate, were not significant for the PRT of drivers.

To compare the differences in the PRT of drivers caused by the influence of the corresponding factors, the probability of not braking pedal can be obtained from the survival function (equation (9)). Figure 4 depicts the estimated probability of not braking pedal for the corresponding covariate when others are controlled for. When the elapsed time has a specific value (e.g., $t=1 \mathrm{sec}$ ), the young participants have a higher probability of not braking pedal compared with the middle-aged ones (see Figure 4(a)); males have an insignificantly higher probability of not braking pedal compared with females (see Figure 4(b)). Cognitive load results in a higher probability of not braking pedal compared with the baseline load (see Figure 4(c)); the probability of not braking pedal increases as the WMC of driver decreases or the initial time headway increases (see Figures 4(e) and 4(f)). However, the lead vehicle's deceleration rate does not lead to a significant change in the probability of not braking pedal (see Figure 4(d)).

\section{Discussion}

The present study investigated the effect of driving situations and individual differences on the PRT of drivers by analyzing the braking behaviors in simulated car-following scenarios. Participating drivers were exposed to different levels of secondarily cognitive load and situational urgency when driving, as well as the WMC of drivers by measuring AOSPAN score, and factors related to driver demographics were considered as explanatory variables for developing the models. The preliminary analysis showed that the PRT of drivers increased with secondarily cognitive load, but a slight change was induced by the initial time headway or deceleration rate of the lead vehicle. To further investigate this effect, several AFT duration models with different types of distributions were developed to model the PRT of drivers. The results indicated that situational factors and individual difference were associated with drivers' braking behavior. The Weibull AFT model with shared frailty is suitable to model the PRT of drivers and was proven to enable better estimation of driver perception response times compared to other duration models.

The models suggested that the presence of secondarily cognitive load while driving was one of the crucial factors impairing the PRT of the drivers in car-following events. Secondarily cognitive load resulted in a $22.6 \%$ increment in the PRT of the drivers for a sudden braking event of the lead vehicle compared with the baseline load. Consistently, the probabilities of not braking pedal were significantly higher for the drivers in distracted states compared to their normal state for the same PRT value. The reduction in the ability to detect the state of the lead vehicle on the road is of the utmost importance for the safety of the driver and other road users. These results matched the study of Bellinger [4] who reported a significant effect of cellular telephone conversations on the PRT of drivers in car-following events. This paper provided further evidence in the impairment of the ability to respond to the lead vehicle's braking scenario by secondarily cognitive load when unobserved heterogeneity is considered.

Previous studies indicated that the time headway and lead vehicle's deceleration rate affected PRT $[2,38]$. Along with these results, the initial time headway was found to significantly affect the PRT, and the probabilities of not braking pedal increased with the initial time headway. This suggests that the initial time headway as a looming cue played a central role for the drivers' response to a breaking lead vehicle. However, in the proposed model, only moderate change in the PRT value was found for $0.6 \mathrm{~g}$ of lead vehicle deceleration rate compared with $0.3 \mathrm{~g}$ of lead vehicle deceleration rate, which was inconsistent with previous studies that pointed out that the increases in the lead vehicle's deceleration rate resulted in a decreased PRT value [2]. There are two reasons that may explain this difference. On the one hand, these previous studies neglected the effect of unobserved heterogeneity on the PRT of drivers; thus, the response to the change in lead vehicle's deceleration rate would differ among individuals. In the present study, this effect was considered properly by constructing the Weibull AFT model with shared heterogeneity. On the other hand, these previous studies did not consider individual factors in the investigation of the driving situation effect on the PRT, which may lead to an overestimation of the influence of driving situations. The introduction of both driving situations and individual differences in the present study would more accurately enable the assessment of the effect of different potential factors. However, more lines of evidence should be provided in future researches related to real driving.

Louie and Mouloua [17] found that the impairing effect of cognitive distraction was more pronounced for individuals with low WMC compared with those with high WMC, suggesting that WMC could play a moderating role on the influence of cognitive distraction on the braking response time. Similarly, the results of the present study demonstrated that WMC exerted a significant effect on the PRT, and the hazard rate decreased by more than $20 \%$ for individuals with higher WMC compared with individuals with the bottom WMC. However, WMC did not lead to a linear improvement of the PRT, and almost identical effects were presented for three individuals with larger WMC. One possible explanation of this result is that the driving task adopted in the present study did not induce higher cognitive demands so that all of these three larger WMC individuals could have sufficient working memory resources to process additional information. However, the influencing mechanisms of working memory capacity on human behaviors remain 


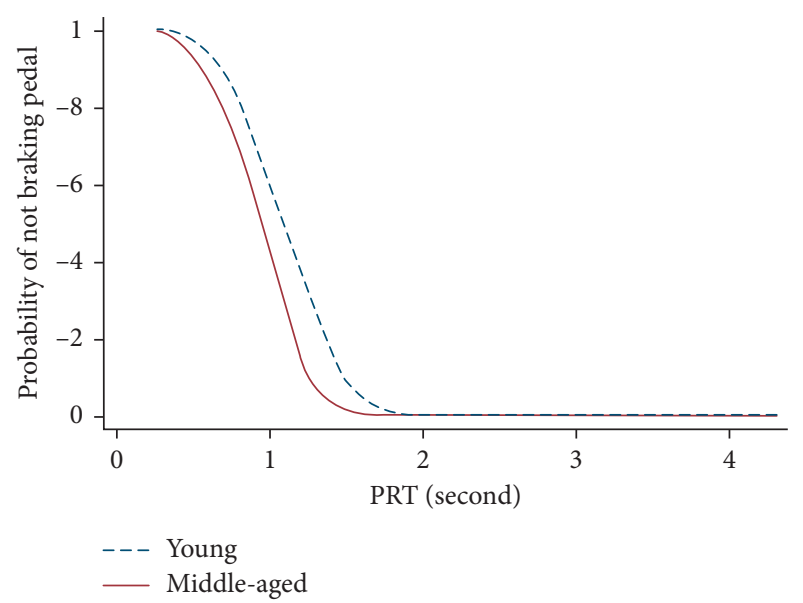

(a)

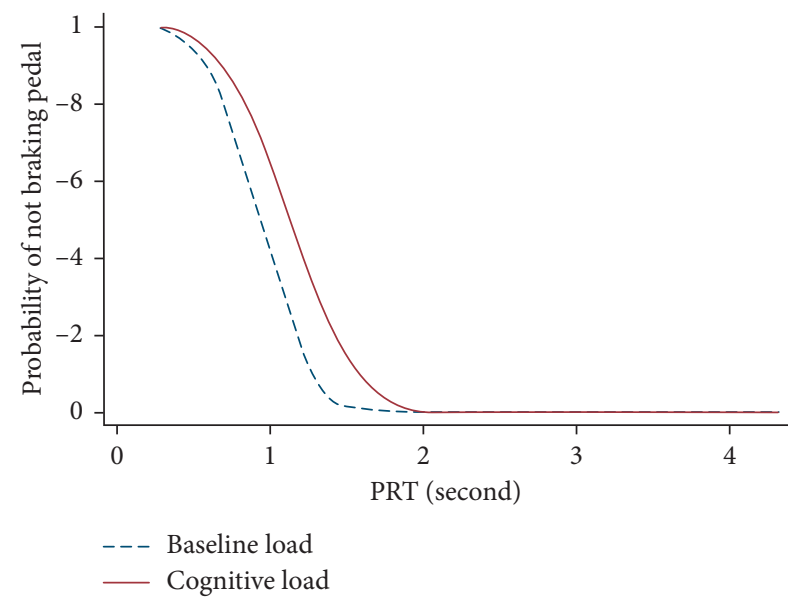

(c)

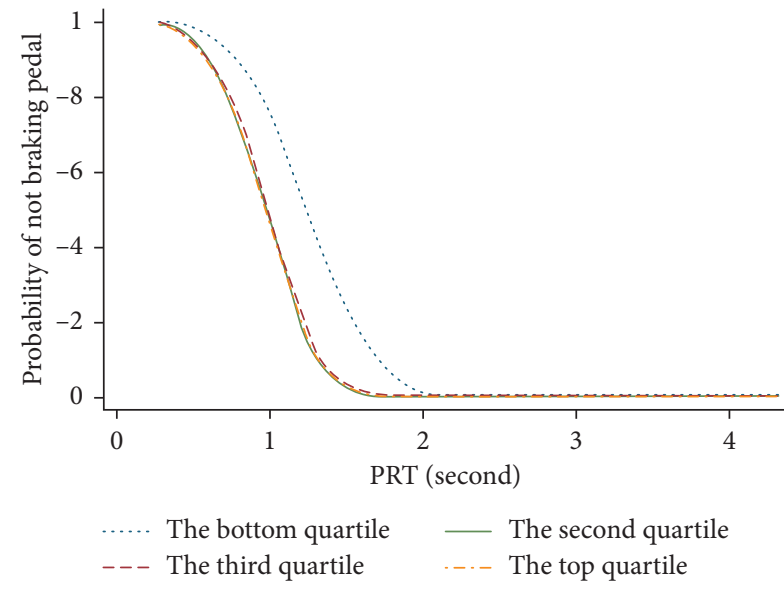

(e)

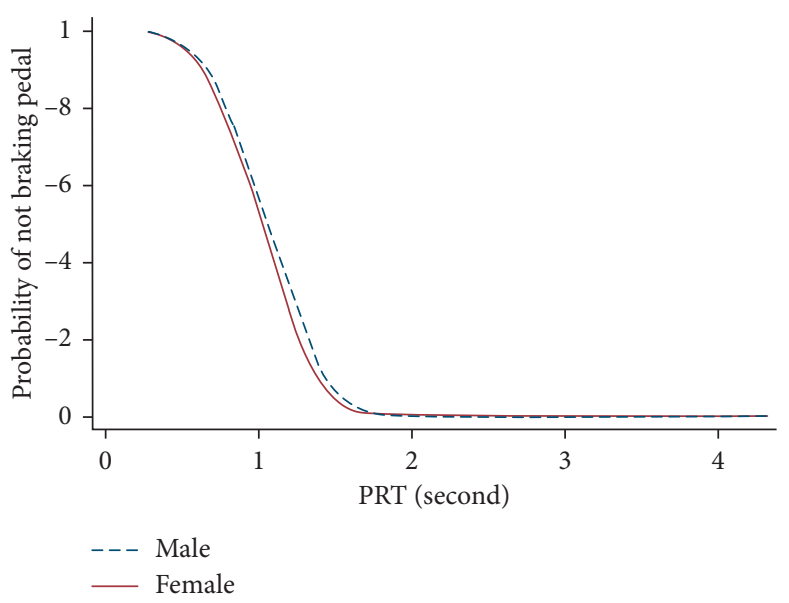

(b)

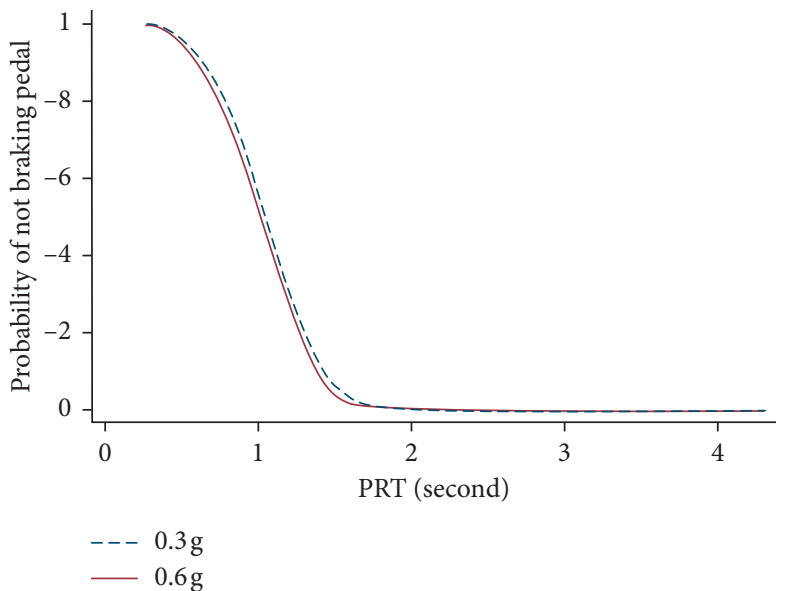

(d)

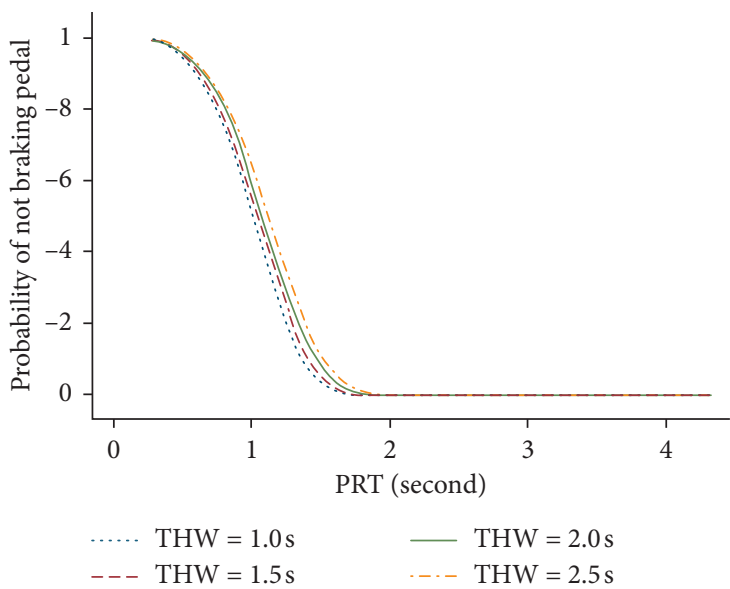

(f)

Figure 4: Comparisons of probability of not braking pedal for (a) young and middle-aged drivers; (b) male and female drivers; (c) baseline load and cognitive load; (d) $0.3 \mathrm{~g}$ and $0.6 \mathrm{~g}$ of lead vehicle's deceleration rate; (e) different levels of WMC; (f) $1.0 \mathrm{~s}, 1.5 \mathrm{~s}, 2.0 \mathrm{~s}$, and $2.5 \mathrm{~s}$ of the initial time headway.

poorly understood, although several theoretical hypotheses have been proposed to explain the role of working memory capacity during human information processing. For example, the n-back task used here has been considered as a complex span task (including processing and storage subtask) and can be driven by an attention control mechanism [39]. Therefore, the degree to the working memory mechanisms driven by different types of driving tasks should be further explored by follow-up studies. Additionally, mature individuals were able to detect the lead vehicle's 
braking event faster than young individuals and, thus, the hazard rate decreased by about $14 \%$ for mature individuals compared with young individuals. However, this difference was not significant between genders. This is consistent with the result of a previous study [40], which suggests that, compared with older drivers, younger drivers demonstrated lower risk aversion and higher propensity for taking risks. Furthermore, the present study provides evidence that age contributes significantly to the impairment in the PRT and leads to a higher driving risk for young drivers in car-following events. This finding is of crucial importance because it suggests that a training intervention aimed at improving young drivers' PRT is necessary in an emergency driving scenario.

In summary, the present study provides further evidence that both driving situations and individual differences affect the PRT of drivers in a lead vehicle's braking event, while considering unobserved heterogeneity. However, the present study faced several limitations. The first problem that must be noted is the unbalanced gender of individuals. Even though previous literature suggested that its impact would be minimal in the study of drivers' reaction time [22], more lines of evidence from female samples are necessary in the future work. Second, the WMC of drivers needs to be assessed by using more tasks, such as operation span task, rotation span task, symmetry span task, and reading span task [12]. A composite measure can help to create a more reliable assessment, since these four tasks can load onto the working memory according to the domain-general model [9]. Third, the results of the present study illustrated that the introduction of unobserved heterogeneity to the proposed model is necessary which implies that there are still other risk factors that have not been included but may affect the PRT of drivers. It is therefore conceivable that more potential influencing factors need to be considered to increase the accuracy of the estimations of subsequent studies. Finally, whether the findings of the present study can be generalized to real driving remains to be verified in future research.

\section{Conclusion}

This study developed AFT duration models to investigate the effect of driving situations and individual differences on the PRT of drivers in simulated car-following events while considering the influence of unobserved heterogeneity. The results showed that the Weibull AFT model with shared frailty can yield a better estimation of the PRT of drivers than other duration models. Furthermore, the influential factors including age, working memory capacity, cognitive load, and initial time headway exhibit a significant effect on the PRT of drivers. The hazard rate changed by $14.4 \%, 22.6 \%$, and $7.5 \%$ when age, cognitive load, and initial time headway changed by one unit, respectively; and the hazard rate decreased by more than $20 \%$ for individuals with higher WMC compared with baseline individuals. One main contribution of this study is the separate identification of the effects of various observed factors on the PRT of drivers while considering the influence of unobserved heterogeneity. These findings provide a better understanding of drivers' braking behaviors, and more accurate estimation of the PRT of drivers can contribute to the development of advanced driving assistant systems or the safety assessment of in-vehicle information and communication technologies.

\section{Data Availability}

The data used to support the findings of this study are available from the corresponding author upon request.

\section{Conflicts of Interest}

The authors declare that there are no conflicts of interest regarding the publication of this paper.

\section{Acknowledgments}

This work was supported by National Key R\&D Program of China (2019YFB1600500), the National Natural Science Foundation of China (51775053), the Changjiang Scholars and Innovative Research Team in University (IRT_17R95), and the Special Research Project of Education Department of Shaanxi Province (19JK0788).

\section{References}

[1] World Health Organization, Global Status Report on Road Safety, World Health Organization, Geneva, Switzerland, 2018, https://www.who.int/violence_injury_prevention/road_ safety_status/2018/en/.

[2] X. Wang, M. Zhu, M. Chen, and P. Tremont, “Drivers' rear end collision avoidance behaviors under different levels of situational urgency," Transportation Research Part C: Emerging Technologies, vol. 71, pp. 419-433, 2016.

[3] S. Y. Sohn and R. Stepleman, "Meta-analysis on total braking time," Ergonomics, vol. 41, no. 8, pp. 1129-1140, 1998.

[4] D. B. Bellinger, B. M. Budde, M. Machida, G. B. Richardson, and W. P. Berg, "The effect of cellular telephone conversation and music listening on response time in braking," Transportation Research Part F: Traffic Psychology and Behaviour, vol. 12, no. 6, pp. 441-451, 2009.

[5] Z. Zhang, Y. Guo, W. Yuan, and C. Wang, "The impact of cognitive distraction on driver perception response time under different levels of situational urgency," IEEE Access, vol. 7, pp. 184572-184580, 2019.

[6] M. L. Aust, J. Engström, and M. Viström, "Effects of forward collision warning and repeated event exposure on emergency braking," Transportation Research Part F: Traffic Psychology and Behaviour, vol. 18, pp. 34-46, 2013.

[7] J. M. Watson and D. L. Strayer, "Supertaskers: profiles in extraordinary multitasking ability," Psychonomic Bulletin \& Review, vol. 17, no. 4, pp. 479-485, 2010.

[8] J. Engström, G. Markkula, T. Victor, and N. Merat, "Effects of cognitive load on driving performance: the cognitive control hypothesis," Human Factors: The Journal of the Human Factors and Ergonomics Society, vol. 59, no. 5, pp. 734-764, 2017.

[9] R. W. Engle, "Working memory capacity as executive attention," Current Directions in Psychological Science, vol. 11, no. 1, pp. 19-23, 2002. 
[10] R. W. Engle and D. Z. Hambrick, "Working memory in the wild: an introduction to the special issue," Journal of Applied Research in Memory and Cognition, vol. 4, no. 5, p. 359, 2016.

[11] G. A. Brewer, B. Hunter Ball, and J. M. Ware, "Individual differences in working memory capacity and shooting behavior," Journal of Applied Research in Memory and Cognition, vol. 5, no. 2, pp. 185-191, 2016.

[12] E. A. Christopher and J. T. Shelton, "Individual differences in working memory predict the effect of music on student performance," Journal of Applied Research in Memory and Cognition, vol. 6, no. 2, pp. 167-173, 2017.

[13] M. A. Pollard and M. L. Courage, "Working memory capacity predicts effective multitasking," Computers in Human Behavior, vol. 76, pp. 450-462, 2017.

[14] K. R. Johannsdottir and C. M. Herdman, "The role of working memory in supporting drivers' situation awareness for surrounding traffic," Human Factors: The Journal of the Human Factors and Ergonomics Society, vol. 52, no. 6, pp. 663-673, 2010.

[15] V. Ross, E. M. M. Jongen, W. Wang et al., "Investigating the influence of working memory capacity when driving behavior is combined with cognitive load: an LCT study of young novice drivers," Accident Analysis \& Prevention, vol. 62, pp. 377-387, 2014.

[16] G. Wood, G. Hartley, P. A. Furley, and M. R. Wilson, "Working memory capacity, visual attention and hazard perception in driving," Journal of Applied Research in Memory and Cognition, vol. 5, no. 4, pp. 454-462, 2016.

[17] J. F. Louie and M. Mouloua, "Predicting distracted driving: the role of individual differences in working memory," $A p$ plied Ergonomics, vol. 74, pp. 154-161, 2019.

[18] M. Green, “"How long does it take to stop?" methodological analysis of driver perception-brake times," Transportation Human Factors, vol. 2, no. 3, pp. 195-216, 2000.

[19] J. W. Muttart, "Estimating driver response times," in Handbook of Human Factors in Litigation, pp. 422-454, CRC Press, Boca Raton, FL, USA, 2005.

[20] S. P. Washington, M. G. Karlaftis, and F. L. Mannering, Statistical and Econometric Methods for Transportation Data Analysis, Chapman \& Hall/CRC, Boca Raton, FL, USA, 2nd edition, 2011.

[21] M. M. Haque and S. Washington, "A parametric duration model of the reaction times of drivers distracted by mobile phone conversations," Accident Analysis \& Prevention, vol. 62, pp. 42-53, 2014.

[22] P. Choudhary and N. R. Velaga, "Modelling driver distraction effects due to mobile phone use on reaction time," Transportation Research Part C: Emerging Technologies, vol. 77, pp. 351-365, 2017.

[23] A. K. Yadav and N. R. Velaga, "Modelling the relationship between different blood alcohol concentrations and reaction time of young and mature drivers," Transportation Research Part F: Traffic Psychology and Behaviour, vol. 64, pp. 227-245, 2019.

[24] Y. C. Li, N. N. Sze, S. C. Wong, W. Yan, K. L. Tsui, and F. L. So, "A simulation study of the effects of alcohol on driving performance in a Chinese population," Accident Analysis and Prevention, vol. 95, pp. 33-342, 2016.

[25] N. Unsworth, R. P. Heitz, J. C. Schrock, and R. W. Engle, "An automated version of the operation span task," Behavior Research Methods, vol. 37, no. 3, pp. 498-505, 2005.

[26] R. J. Nowosielski, L. M. Trick, and R. Toxopeus, "Good distractions: testing the effects of listening to an audiobook on driving performance in simple and complex road environments," Accident Analysis \& Prevention, vol. 111, pp. 202-209, 2018.

[27] P. D'Addario and B. Donmez, “The effect of cognitive distraction on perception-response time to unexpected abrupt and gradually onset roadway hazards," Accident Analysis \& Prevention, vol. 127, pp. 177-185, 2019.

[28] N. Wildwall, M. Falkenstein, and P. D. Gajewski, “Age-related differences in working memory performance in a 2-back task," Frontiers in Psychology, vol. 2, p. 186, 2011.

[29] L. Lilienthal, E. Tamez, J. T. Shelton, J. Myerson, and S. Hale, "Dual $n$-back training increases the capacity of the focus of attention," Psychonomic Bulletin \& Review, vol. 20, no. 1, pp. 135-141, 2013.

[30] P. Van den Berg, T. Arentze, and H. Timmermans, "A latent class accelerated hazard model of social activity duration," Transportation Research Part A: Policy and Practice, vol. 46, no. 1, pp. 12-21, 2012.

[31] X. Liu, Survival Analysis: Models and Applications, John Wiley \& Sons, Hoboken, NJ, USA, 2012.

[32] M. Cleves, W. Gould, W. W. Gould, R. G. Gutierrez, and Y. Marchenko, An Introduction to Survival Analysis Using Stata, Stata Press, College Station, TX, USA, 2nd edition, 2008.

[33] X. Yang, M. Abdel-Aty, M. Huan, Y. Peng, and Z. Gao, “An accelerated failure time model for investigating pedestrian crossing behavior and waiting times at signalized intersections," Accident Analysis \& Prevention, vol. 82, pp. 154-162, 2015.

[34] D. Nam and F. Mannering, "An exploratory hazard-based analysis of highway incident duration," Transportation Research Part A: Policy and Practice, vol. 34, no. 2, pp. 85-102, 2000.

[35] A. T. Hojati, L. Ferreira, S. Washington, and P. Charles, "Hazard based models for freeway traffic incident duration," Accident Analysis \& Prevention, vol. 52, pp. 171-181, 2013.

[36] H. Akaike, "A new look at the statistical model identification," IEEE Transactions on Automatic Control, vol. 19, no. 6, pp. 716-723, 1974.

[37] G. Schwarz, "Estimating the dimension of a model," The Annals of Statistics, vol. 6, no. 2, pp. 461-464, 1978.

[38] Q. Xue, G. Markkula, X. Yan, and N. Merat, "Using perceptual cues for brake response to a lead vehicle: comparing threshold and accumulator models of visual looming," Accident Analysis \& Prevention, vol. 118, pp. 114-124, 2018.

[39] Z. Shipstead, D. R. B. Lindsey, R. L. Marshall, and R. W. Engle, "The mechanisms of working memory capacity: primary memory, secondary memory, and attention control," Journal of Memory and Language, vol. 72, pp. 116-141, 2014.

[40] J. Hatfield and R. Fernandes, "The role of risk-propensity in the risky driving of younger drivers," Accident Analysis \& Prevention, vol. 41, no. 1, pp. 25-35, 2009. 